\title{
A Novel Adjacent Sensors-Based Mechanism to Increase Performance of Wireless Sensor Networks
}

\author{
Mohammed Al-Shalabi (iD, Jafar Ababneh, and Waled Abdulraheem \\ Faculty of Information Technology, The World Islamic Sciences and Education University, Amman, Jordan \\ Correspondence should be addressed to Mohammed Al-Shalabi; mohammed.shalabi@wise.edu.jo
}

Received 27 December 2020; Revised 1 March 2021; Accepted 22 March 2021; Published 29 March 2021

Academic Editor: Mauro Parise

Copyright ( 2021 Mohammed Al-Shalabi et al. This is an open access article distributed under the Creative Commons Attribution License, which permits unrestricted use, distribution, and reproduction in any medium, provided the original work is properly cited.

\begin{abstract}
Wireless Sensor Networks are widely used nowadays to support the decision-makers in different applications by monitoring and collecting the environmental parameters in specific areas. Sensors are deployed in such areas either randomly or formally. In a high-density Wireless Sensor Network, several sensors are randomly deployed in a small area. This will make the adjacent sensors collect same data and send them to the sink, which will increase the power consumption in those sensors. Adjacent sensors are considered critical because of their effect on the network performance. In this paper, the effect of the adjacent sensors is minimized because of the above-mentioned criticality and performance influence of these sensors. The proposed mechanism is evaluated by using MATLAB simulator and is then compared with the low-energy adaptive clustering hierarchy (LEACH) protocol. Results prove that the proposed mechanism outperforms the LEACH protocol by $21 \%$ in terms of the network lifetime and by $18 \%$ in terms of the number of the transmitted packets to the cluster heads and reduces the number of the transmitted packets to the base station by approximately $3 \%$ by avoiding the duplicated packets.
\end{abstract}

\section{Introduction}

Wireless Sensor Network (WSN) is an important field in the information technology and is used to sense the critical environmental parameters in many applications where the humans are difficult to sense, such as underwater monitoring, fire expectation monitoring, and humidity monitoring $[1,2]$.

WSNs consist of many sensors that sense the critical parameters in a specific area and transmit the values to the base station (BS) for further analysis [3]. In clustering WSNs, the area is divided into clusters; each cluster has many sensors and one specific sensor called cluster head $(\mathrm{CH})$. The $\mathrm{CH}$ collects the data from other sensors in its corresponding cluster, aggregate it, and then send the aggregated data to the BS [4].

Routing is an important criterion when designing WSNs because of their applicability over wide areas. Routing protocols for WSNs are categorized into clustering, locationbased, and flat routing protocols depending on the network structure [3]. Clustering routing protocols have been a major research topic as a result of their advantages in distributing energy among sensors and prolonging the overall lifetime of a network [5]. An example of basic clustering routing protocols is the low-energy adaptive clustering hierarchy (LEACH) [5] protocol.

LEACH protocol increases the network lifetime by distributing the energy in all the sensors by rotating the task of the $\mathrm{CH}$ among all the sensors in the network $[6,7]$. The $\mathrm{CH}$ possesses additional duties, such as collecting, gathering, and transmitting data. Thus, it consumes more energy than other normal sensors. However, LEACH protocol suffers from many drawbacks, such as the randomness in selecting $\mathrm{CHs}$ without considering the residual energy of the sensors and the distance neither among them nor between a $\mathrm{CH}$ and the BS [8].

Some applications require a large number of sensors to be randomly deployed in a small area, which increases the density of the sensors. Increasing the density of the sensors causes some of them to be very close to each other and share 
the same sensing area. In such case, duplicated packets will be transmitted to a $\mathrm{CH}$, which causes the adjacent sensors and the $\mathrm{CH}$ to consume more energy in sensing, aggregating, and transmitting these packets to the BS.

In this paper, an enhanced mechanism of LEACH protocol is proposed to deal with the adjacent sensors and prevent them from sensing the same data. Accordingly, the consumed energy in each sensor can be minimized, thereby maximizing the lifetime of the sensor and the entire network. Moreover, an equation is proposed to determine the shortest distance between sensors, which will be considered by the BS. In a nutshell, the contributions of this paper are as follows:

(1) Utilizing the distance between the sensors in the hierarchal-based WSNs efficiently to prolong the lifetime of the network

(2) Formulating an equation to define the distance between the sensors

The remaining parts of the paper are structured as follows. Section 2 presents a background on LEACH protocol. Section 3 provides a summary of the related works. Section 4 presents the adjacent sensors' problem. Section 5 explains the proposed mechanism in detail. Section 6 provides a quantitative comparison between the proposed mechanism and LEACH protocol, and Section 7 elaborates the conclusions of the paper.

\section{Background of LEACH Protocol}

The hierarchal protocols in WSNs aim to maximize the network lifetime by forming a set of clusters which contains several sensors in each cluster in addition to the $\mathrm{CH}$ [9]. These protocols also aim to minimize the number of packets that will be sent to the BS by using the data aggregation in a single sensor, which is a $\mathrm{CH}$, inside a cluster instead of transmitting a packet directly to the BS from each sensor [10]. Figure 1 illustrates the idea of the hierarchical protocols $[6,10]$.

Some of the hierarchical protocols that have been proposed for the sensor networks are LEACH [5], power-efficient gathering in the sensor information system [11], threshold-sensitive energy-efficient sensor network [12], and the adaptive TEEN [13]. The most popular protocol, which is the LEACH protocol, in addition to many variants of it, is presented in Section 3. LEACH protocol is explained in detail in the following subsection, since it is a popular protocol in the hierarchy-based WSNs. The proposed mechanism enhances it, in addition to explaining its performance for comparison purposes with the proposed mechanism.

2.1. LEACH Routing Protocol. LEACH protocol is the first energy-efficient clustering protocol that has been presented [5] to enhance the efficiency of WSNs by increasing their lifetime. Using LEACH protocol, sensors are grouped in several groups called clusters. A central device called BS conducts further analysis of sensing the data. The sensors

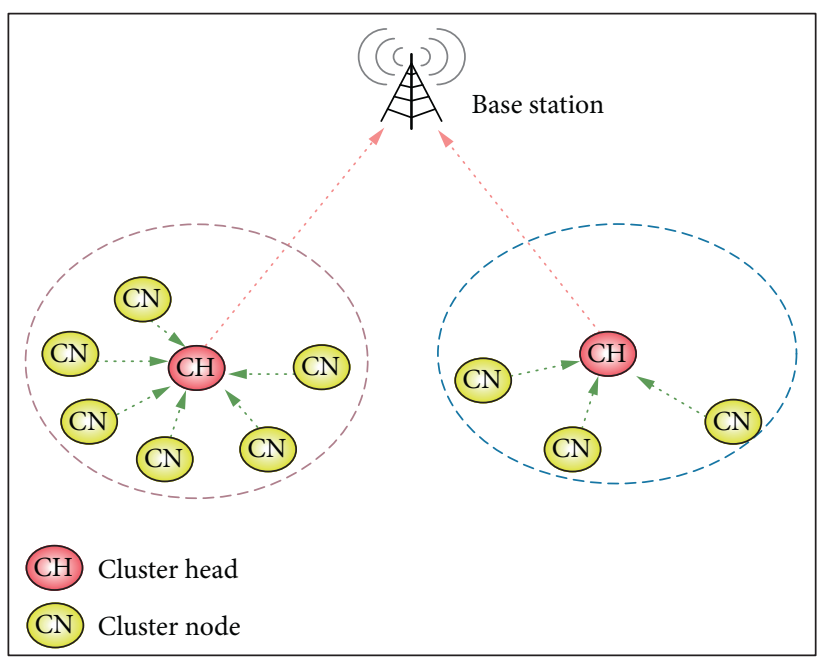

FIGURE 1: Hierarchical-based routing protocols.

inside each cluster are divided into two types: a set of sensors for sensing purposes and a sensor called $\mathrm{CH}$. The $\mathrm{CH}$ collects data from other sensors and then sends them to the BS [14].

LEACH protocol consists of two stages, namely, the setup and steady state. In the setup phase, the sensors in each cluster create random numbers between 0 and 1 in each round. A reference number $T(n)$ is predefined for comparison with the created numbers. When the reference number is greater than the randomly created number, the sensor that creates the random number becomes the $\mathrm{CH}$. Equation (1) is used to determine the reference number $T(n)$ [15].

$$
T(n)= \begin{cases}\frac{P}{1-P *(r \bmod 1 / P)}, & n \in G, \\ 0, & n \in G,\end{cases}
$$

where $P$ is the percentage of CHs, $r$ is the existing round, and $G$ is a group of sensors which did not become a $\mathrm{CH}$ in the prior $1 / P$ rounds [15].

Sensors that are CHs will send a message to all the other sensors, and then each sensor will select the adequate $\mathrm{CH}$ depending on the strength of a signal. When a sensor selects its potential $\mathrm{CH}$, it transmits a message to it to be a member of a cluster. Thereafter, the clusters form, and the $\mathrm{CH}$ creates a time division multiple access table to avoid data collision during transmission. Finally, the $\mathrm{CH}$ sends this timetable to other member sensors in its cluster [16].

During the steady-state stage, each $\mathrm{CH}$ collects the data, which are sent by the sensors in its related cluster, and transmits them to the BS [17]. Figure 2 describes the structure of the steady-state phase.

LEACH protocol exhibits many advantages, such as maximizing the network lifetime by distributing the energy among sensors in a network. However, this protocol also presents many disadvantages, such as ignoring the residual energy of sensors when selecting $\mathrm{CHs}$.

LEACH protocol neglects the distance between sensors in the sensing process, especially in the case of the randomly 


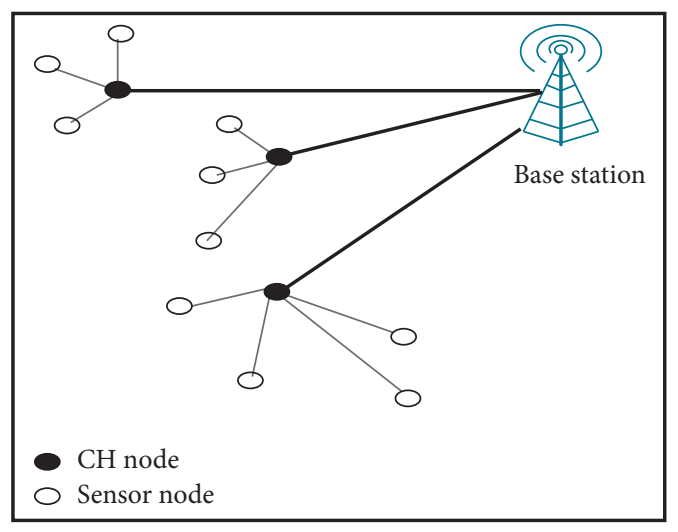

Figure 2: Structure of the steady-state phase.

deployed sensors in a sensing region of a high-density network. In this case, the probability of adjacent sensors increases. This problem directly impacts the lifetime performance of adjacent sensors. As a result, the lifetime efficiency of the entire network decreases.

According to the above-mentioned explanations, several routing protocols have been proposed to enhance the performance of LEACH protocol by maximizing the network lifetime. These protocols are explained in the following section.

\section{Related Works}

Some routing protocols that are based on LEACH and related to the proposed mechanism are presented in this section. The following routing protocols have been presented to overcome the limitation of LEACH in considering the distance between the sensors in the sensing process.

The authors in [18] presented a mechanism called areaaware coverage to minimize the overlapping coverage area among the sensors of variable radii. The mechanism in [18] finds the redundant sensors by allowing each sensor to calculate its coverage area and find the number of shared sensors within it. Then, the sensor will adjust its radius depending on the predefined threshold. A sensor will be turned off if its radius is less than the radius threshold, and this way is insufficient as a result of the effect on the network coverage. This mechanism reduces the active sensors by turning off the duplicate sensors depending on the distance between them. If the distance from sensor " $a$ " to sensor " $b$ " is less than or equal to the radius of sensor " $a$," then sensor " $b$ " will be turned off. This way also affects the network coverage negatively because some uncovered points between the sensors still exist if one of them is turned off depending only on the radius. The mechanism presents many limitations according to what is mentioned above; in addition, this mechanism works with sensors of an adjustable radius value.

The authors in [19] suggested a mechanism to increase the lifetime of a network by dividing the network into two groups depending on the distance between sensors. The two groups alternate in the sensing process. The mechanism in [19] is limited by its weak dividing process, which depends only on the distance among sensors without considering the overlapping among them. The sensors, which are close to each other, will be classified into two groups even if they do not overlap. This way impacts negatively the network coverage due to the alternating sensing process.

The authors in [20] proposed a mechanism to minimize the data redundancy by finding the overlapped area of a sensor on the basis of the nearby sensors. The mechanism in [20] finds the total overlapped area, which is common among three circles. After finding the overlapping sensing area by the neighbors of each sensor in a network, the redundant sensors will deactivate depending on a predefined threshold. A sensor of an overlapping sensing area more than the predefined value will prompt its neighbors to run sleep mode. If all the neighboring sensors send permission to this sensor, then the sensor will be in sleeping mode. This mechanism increases the lifetime of the network but possesses high complexity because of the calculations for finding the total overlapped area. The mechanism also ignores the overlapping problem in the entire network, as it only considers the overlapping among three circles. The authors did not use the technique to change the redundant sensors from the sleeping mode to active mode.

The clustering mechanism in [21] maximizes the lifetime of a network by identifying the overlapped $\mathrm{CHs}$ and using them in the routing process. The mechanism in [21] uses a layered approach to form clusters. Thereafter, the CHs will be identified by finding the distance from each sensor to the center of the cluster. Then, a sensor with the minimum distance to the center will be a $\mathrm{CH}$ of that cluster. After identifying the $\mathrm{CH}$ s for each cluster, the overlapped $\mathrm{CH}$ s will be identified as the sensors that are near the $\mathrm{CH}$ and their cluster boundary. This mechanism uses the bottom-top approach to transfer the data from a $\mathrm{CH}$ to the BS through a set of other $\mathrm{CH}$ and overlapped CHs. The mechanism ignores the overlapping among sensors in each cluster and cannot minimize the effect of overlapping.

The authors in [22] proposed a common sensor exploitation approach. In this approach, the entire region is divided into several grids. The $\mathrm{CH}$ s will be positioned approximately at the intersection points of each grid. The sensors, which are located in the common area between the clusters, can directly select any of the overlapping $\mathrm{CHs}$ depending on the distance from it to the BS, and these sensors then communicate with the $\mathrm{CH}$ directly to select the shortest path to the BS. The mechanism in [22] ignores the distance between the sensors in the entire network but focuses on the sensors in the overlapped area of the adjacent clusters.

The authors in [23] proposed an awareness coverage mechanism for WSNs to schedule the sleeping of the sensors based on the overlapped area with the adjacent sensors. The mechanism in [23] considers the increase in the sleeping sensors while minimizing the active sensors to increase the network lifetime. When the overlapped sensors are identified, the sensors of equal sensing range will sleep while keeping one sensor in the active mode. This mechanism does not produce a clear sleeping schedule and does not use a clustering approach. 
From the above-mentioned review of the related works, no proposed mechanism is found to consider the LEACH protocol as the basic clustering protocol in addressing its limitation. Most of the previously proposed mechanisms concentrated on the distance among the clusters rather than that of the sensors. In addition, the related mechanisms considered the distance between the used sensors in different techniques such as increasing the active sensors or grouping the sensors without any consideration of the coverage area of these sensors.

In a nutshell, there is a need for a comprehensive mechanism that utilizes the distance between the adjacent sensors in an efficient way to prolong the network lifetime by identifying these sensors based on a proposed equation to increase the efficiency of the proposed mechanism.

The currently proposed mechanism enhances the LEACH protocol to increase the efficiency of the clustering based WSNs in terms of network lifetime and power consumption by utilizing the distance between the sensors. Moreover, the proposed mechanism overcomes the problems related to the adjacent sensors by formulating an equation to determine the distance between them. The adjacent sensors are explained in detail in the following section.

\section{Distance between Sensors}

In a high-density WSN, several sensors are randomly deployed. Thus, the possibility of two sensors being close to each other increases. If two sensors are close to each other, then these sensors share approximately the same sensing area. These sensors are called adjacent sensors, and there is a shared area between them (Figure 3).

As noted in Figure 3, two adjacent sensors ( $s 1$ and $s 2$ ) share approximately the sensing region, based on the sensing range of the sensors, and send the same data $(v 1, v 2$, and $v 3)$ to the $\mathrm{CH}$. This situation leads to fast draining of energy in the adjacent sensors and their sudden death. Moreover, the aggregation process and the transmission process of the duplicated data in the $\mathrm{CH}$ make it consume much energy. All these reasons impact the lifetime of the adjacent sensors and thus the entire network.

A major problem occurs when the distance between two sensors $(d)$ is small enough, which causes the interference of the radiuses of the sensing areas $(r 1$ and $r 2)$ of the two sensors. The sensors ( $s 1$ and $s 2)$ will sense the same data and send the same values $(v 1, v 2$, and $v 3)$, which are located in the shared area, to the $\mathrm{CH}$. The proposed clustering mechanism that utilizes the distance between adjacent sensors is discussed in detail in the following section.

\section{Proposed Mechanism}

In the previous sections, LEACH protocol is discussed in detail because it is considered the main cluster-based hierarchal protocol. It suffers from many limitations particularly in terms of distance among the sensors and dealing with the adjacent sensors. Many mechanisms have been

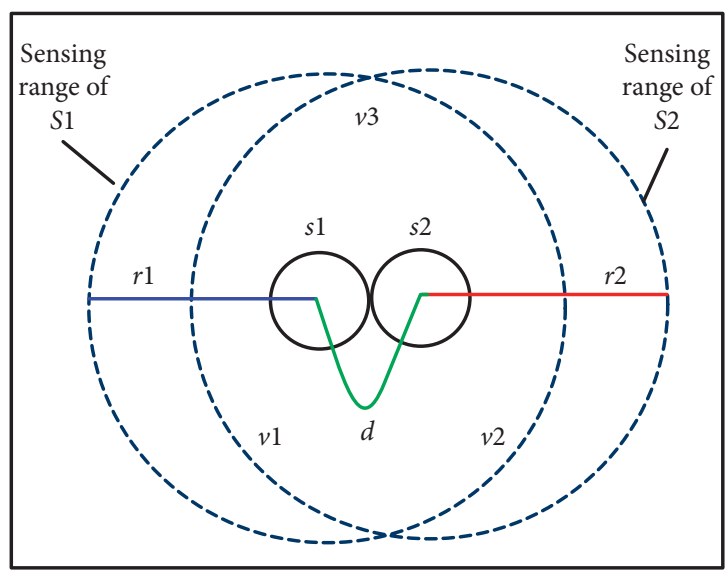

FIgURE 3: Shared area between two adjacent sensors.

presented to enhance the LEACH protocol, but these mechanisms did not utilize the distance between the sensors.

As discussed earlier, the adjacent sensors strongly affect the lifetime of the corresponding $\mathrm{CHs}$, thereby affecting the lifetime of the network. The negative effect of the adjacent sensors should be reduced as much as possible to increase their lifetime as long as possible, thus solving the abovementioned issues.

The proposed mechanism satisfies these conditions and hence can prolong the network lifetime while maintaining the network coverage.

In the proposed clustering mechanism, many sensors are randomly deployed in a region; then these sensors are grouped in the clusters using the same procedure in LEACH protocol.

Firstly, the BS uses the locations of the sensors to identify the distance between them and defines the adjacent sensors based on a predefined threshold. Clusters are formed with a $\mathrm{CH}$ for each cluster using the same procedure in the $\mathrm{LEACH}$ protocol. At the same time, the sensors follow the same phases in the LEACH protocol to form the clusters and select the CHs. Figure 4 shows the structure of the proposed clustering mechanism.

As shown in Figure 4, the BS identifies the adjacent sensors based on the distance between them and the predefined threshold. If the distance between two sensors is equal to or less than the threshold, then the BS considers them as adjacent sensors such as the sensors in red and yellow colors in Figure 4.

The sensing operation of the adjacent sensors should be organized by the BS to reduce the redundancy of the sensed data. Therefore, defining the threshold distance between the sensors is important to organize the sensing and transmission operations.

As mentioned earlier, the sensors are initially distributed randomly in a specific region. Then, the BS identifies the adjacent sensors depending on the distance between them and by using the radius $r$ of the sensing area of each sensor. The idea is to utilize the adjacent sensors and manage the sensing process of each sensor to maintain their energy as much as possible. Moreover, the idea is to deal with the adjacent sensors ( $s 1$ and $s 2$ ) separately from each other by 


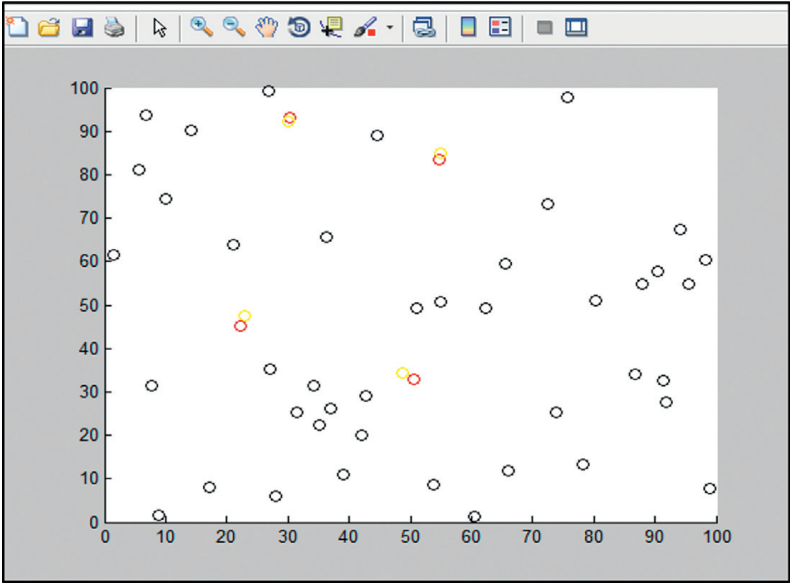

FIGURE 4: Structure of the proposed clustering mechanism.

making one of them $(s 1)$ sense the surrounding parameters, while the other is $(s 2)$ idle. In contrast, when the sensor $(s 1)$ dies due to the expiry of its energy, sensor $(s 2)$ starts sensing the surrounding parameters. In this mechanism, the lifetime of the entire network will increase as much as possible by saving the energy of the adjacent sensors as much as possible and keeping them sense the surrounding parameters for a long time.

The radius $(r)$ of the sensing area is the same for all sensors because they are homogenous. The BS knows the coordinates of each sensor in the network. In this mechanism, the sensors do not know the locations of their neighbors to avoid the additional message overheads and calculations. Only the BS knows the locations of the sensors to define the distance between them and identify the adjacent sensors.

The BS uses a predefined threshold value $T d$ to identify the adjacent sensors as explained in the following equation:

$$
T d \leq r 1+r 2,
$$

where $T d$ is the threshold distance between the centers of two sensors, $r 1$ is the radius of the first sensor, and $r 2$ is the radius of the second sensor. Based on equation (2), the two sensors are considered adjacent if the distance between them is equal to or less than the summation of their radiuses.

The distance between the centers of two sensors is the Euclidean distance between sensor $s 1$ and sensor $s 2$ and is given in the following equation:

$$
d\left(\left(x s_{1}, y s_{1}\right),\left(x s_{2}, y s_{2}\right)\right)=\sqrt{\left(x s_{1}-x s_{2}\right)^{2}+\left(y s_{1}-y s_{2}\right)^{2}},
$$

where $\left(x s_{1}, y s_{1}\right)$ and $\left(x s_{2}, y s_{2}\right)$ are the coordinates of sensors $s_{1}$ and $s_{2}$, respectively. Figure 5 illustrates the adjacent sensors.

Figure 5 shows that the distance between $s 1$ and $s 2$ equals the summation of $r 1$ and $r 2$.

The above-mentioned situation indicates that if the distance between two sensors is equal to or less than $T d$, then the two sensors are adjacent.

The BS will divide the adjacent sensors into two clusters; if the distance from sensor $s 1$ to sensor $s 2$ is less than or equal

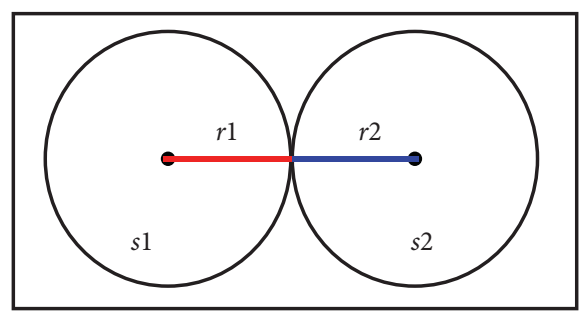

Figure 5: Adjacent sensors.

to $\mathrm{Td}$, then the BS will categorize the two sensors in different clusters; otherwise, the sensors will not be categorized and will be considered normal sensors.

The sensors in the first cluster will start sensing the surrounding parameters, while the sensors in the second cluster are idle. If one sensor from the first cluster dies after consuming its energy, its adjacent sensor from the second cluster starts sensing. This mechanism increases the lifetime of the entire network by saving the energy in the adjacent sensors as much as possible. Figure 6 shows the proposed mechanism.

As noted in Figure 6, sensors $s 1$ and $s 2$ are identified by the BS as adjacent sensors based on the predefined threshold as described in equation (2). Sensor $s 1$ belongs to the first cluster and its status is active and it will start sensing, while sensor $s 2$ belongs to the second cluster and its status is idle. In the proposed mechanism, when the energy of sensor $s 1$ almost finishes, it sends a message to the BS to inform it about the situation. Afterward, the status of sensor $s 2$ will be directly changed by the BS to be active, which indicates that it is an active sensor and will start sensing. This mechanism keeps the sensing coverage of the network stable as much as possible and increases the network reliability.

Next is the algorithm of the proposed mechanism.

The algorithm of the proposed mechanism is as follows:

(1) Sensors are randomly deployed.

(2) The adjacent sensors will be divided by the BS into two clusters as follows: If the distance between sensor $s 1$ and sensor $s 2$ is less than or equal to a predefined threshold as described in equation (2), then sensor $s 1$ will be an active sensor and sensor $s 2$ will be an idle sensor.

(3) Clusters will be formed and a cluster head will be assigned to each cluster using the same way in the LEACH protocol.

(4) The sensors in the first cluster shall start sensing, while the ones in the second cluster are idle.

(5) When the energy of any sensor in the first cluster is almost finished, it sends a message to the BS informing it about the situation.

(6) The BS changes the status of the adjacent sensor in the second cluster to active and it will start sensing.

The simulation results of the proposed mechanism are presented and discussed in the following section as well as the quantitative comparison between the LEACH protocol and the proposed mechanism. 


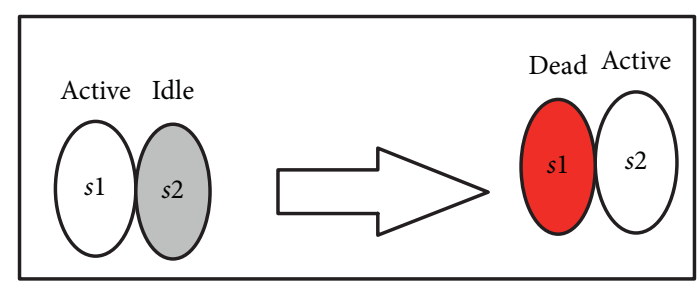

Figure 6: The proposed mechanism.

\section{Simulation Results and Comparison}

In the proposed clustering mechanism, the lifetime of the entire network depends on that of the adjacent sensors. Thus, the lifetime of each sensor must be computed to define the lifetime of the overall network. Following the definition in [24], the remaining lifetime equals the remaining energy divided by the initial energy, as shown in the following equation:

$$
\mathbf{L}_{\mathbf{S}}=\frac{\mathbf{E}_{0}-\sum_{\mathbf{i}=1}^{\mathbf{N}} \mathbf{E}_{\mathbf{T x}}+\mathbf{E}_{\mathbf{R x}}}{\mathbf{E}_{0}}
$$

where $L_{S}$ denotes the lifetime of the sensors; $E_{0}$ is the initial energy; $E_{T x}$ and $E_{R x}$ are the required energy to send and receive the packets, respectively, in a specific round; equations (5) and (6) represent the calculations of both metrics based on the energy model in [25]; and $N$ is the number of rounds.

$$
E_{T x}=E_{\mathrm{elec}} * k+E_{\mathrm{amp}} * k * d^{n}
$$

where $E_{\text {elec }}$ represents the electronic energy, $E_{\mathrm{amp}}$ denotes the energy of the amplifier, and $n$ equals 2 in the free space and equals 4 in the indoor environments [25]. The required energy to receive $k$ bits packet is

$$
E_{R x}=E_{\mathrm{elec}} * k,
$$

In order to simplify the above-mentioned equation in (4), the following condition is considered:

$$
L_{S}=1-\frac{\sum_{i=1}^{N} E_{T x}+E_{R x}}{E_{0}},
$$

Then, a network containing some adjacent sensors is assumed. The process of sensing the data based on the number of the adjacent sensors in the proposed mechanism. Thus, the lifetime of the adjacent sensors in the first cluster is computed in the following equation by modifying equation (7) as follows:

$$
L_{\mathrm{AS} 1}=\left(1-\frac{\sum_{i=1}^{N} E_{T x}+E_{R x}}{E_{0}}\right),
$$

where $L_{\mathrm{AS} 1}$ is the lifetime of the adjacent sensors in the first cluster.

Equation (8) indicates that the lifetime of the adjacent sensors in the first cluster, which are the first active ones, is approximately the same as the lifetime of the normal sensors because they sense together at the same time, while the adjacent sensors in the second cluster are idle.
The lifetime of the adjacent sensors in the second cluster, which start sensing when the adjacent sensors in the first cluster die, is computed in the following equation:

$$
L_{\mathrm{AS} 2}=\left(1-\frac{\sum_{i=1}^{N 1} E_{T x}+E_{R x}}{E_{0}}\right),
$$

where $L_{\mathrm{AS} 2}$ is the lifetime of the adjacent sensors in the second cluster and $N_{1}$ is the number of rounds where the adjacent sensors in the second cluster are active.

Equation (9) indicates that the lifetime of adjacent sensors is greater than the lifetime of the normal sensors because of the increase of the sensing time for the adjacent sensors. Any adjacent sensor in the first cluster will sense data in $N$ rounds before it dies. Then the second adjacent sensor will start sensing in $N 1$ rounds. Thus, the lifetime of the entire network which uses the proposed mechanism is increased by $N 1$ rounds over the lifetime of the entire network which does not use the proposed mechanism. The lifetime of the entire network is computed using the following equation:

$$
L_{\mathrm{NW}}=\sum_{i=1}^{N_{\text {normal }}} L_{\mathrm{S}}(i)+\sum_{j=1}^{N_{\text {adjacent1 }}} L_{\mathrm{AS} 1}(j)+\sum_{w=1}^{N_{\text {adjacent2 }}} L_{\mathrm{AS} 2}(w),
$$

where $L_{\mathrm{NW}}$ is the lifetime of the entire network; $N_{\text {normal }}$ denotes the number of normal sensors in a network; $N_{\text {adjacent1 }}$ represents the adjacent sensors in the first cluster; $N_{\text {adjacent2 }}$ represents the adjacent sensors in the second cluster; $L_{\mathrm{S}}, L_{\mathrm{AS} 1}$, and $L_{\mathrm{AS} 2}$ are the lifetimes of the normal sensors and the adjacent sensors in the two clusters in the network as explained in equations (7)-(9), respectively.

Equation (10) indicates that the lifetime of the adjacent sensors, which are considered critical sensors, will determine the lifetime of the entire network depending on the parameters used in this model. The main metrics that are used to evaluate the proposed mechanism and compare it with LEACH protocol are listed in Table 1.

The comparison is done with the LEACH protocol because the proposed mechanism enhances it.

Table 2 presents the main considered parameters for simulating the proposed mechanism.

The basic parameters and values in Table 2 are based on the previous studies and as proposed in the LEACH protocol [5].

As noted in Table 2, there are a large number of sensors deployed in a small area to comprehensively determine the effect of sensor density. The simulation is conducted ten times to reduce the effect of randomness. Moreover, in order to get a fair comparison, the proposed mechanism and the LEACH protocol are applied on the sensors in the same locations where each one is running. The proposed mechanism is simulated by using MATLAB with the simulation parameters listed in Table 2.

As seen in Table 2, the value of the predefined threshold $(\mathrm{Td})$ is set to 1 in the simulation to make the BS consider only the adjacent sensors for grouping in clusters. Equations (8) and (9) are considered to find the lifetime of the adjacent sensors in the two clusters. 
TABLE 1: Evaluation metrics.

\begin{tabular}{lc}
\hline Metric & Description \\
\hline LND & The last sensor dies (in rounds) \\
Packets to BS & The number of the packets which are sent from the CHs to the BS \\
Packets to CHs & The number of the packets which are sent from the sensors to the CH \\
\hline
\end{tabular}

TABLE 2: Simulation parameters.

\begin{tabular}{lcc}
\hline Symbol & Declaration & Value \\
\hline$A$ & Network size & $100 \times 100$ \\
BS $(i, j)$ & Position of base station & Centered $(50 \times 50)$ \\
$N$ & Number of sensors & 300 \\
$E_{0}$ & Initial energy & $0.5 \mathrm{Joule}$ \\
$E_{\mathrm{amp}}$ & Transmit amplifier & $13 \mathrm{pJ} / \mathrm{bit} / \mathrm{m}^{2}$ \\
$E_{\text {elec }}$ & Electronic energy consumption & $50 \mathrm{~nJ} / \mathrm{bit}$ \\
$E_{r x}$ & Reception energy & $50 \mathrm{~nJ} / \mathrm{bit}$ \\
$E_{t x}$ & Transmission energy & $50 \mathrm{~nJ} / \mathrm{bit}$ \\
$E_{\mathrm{da}}$ & Data aggregation energy & $5 \mathrm{~nJ} / \mathrm{bit}$ \\
$r_{\max }$ & Maximum number of rounds & 10000 \\
$r$ & Radius of each sensor & $0.5($ for \\
$T d$ & Predefined threshold & simulation purposes) \\
\hline
\end{tabular}

In the simulation scenario, the number of sensors is 300 . These sensors will be randomly deployed on the area of $100 \mathrm{~m} \times 100 \mathrm{~m}$ as illustrated in Figure 7 .

Figure 7 shows the adjacent sensors among the 300 deployed sensors in the area. The yellow and red circles denote the adjacent sensors in the network. The red circles denote the sensors belonging to the first cluster, and the yellow circles denote the sensors belonging to the second cluster. The simulation results after applying the proposed mechanism and the LEACH protocol on the network are presented in Figures 8-10.

Figure 8 shows the Last Node Dies (LND) values in each run for the proposed mechanism and the LEACH protocol.

As shown in Figure 8, the average of the LND of the proposed mechanism in the tenth runs is 3409.4 rounds, whereas that of the LEACH protocol is 2825.2 rounds. The results indicate that the proposed mechanism increases the lifetime of the entire network by 584.2 rounds with the approximated percentage of $21 \%$ in all the runs. Moreover, Figure 8 shows that the lifetime of the entire network in the proposed mechanism is more than that in the LEACH protocol in seven runs out of ten, which means that, in approximately $70 \%$ of the runs, the lifetime of the network increases. Figure 9 shows the comparison between the two mechanisms based on the number of the transmitted packets to the BS.

The average number of the transmitted packets from $\mathrm{CHs}$ to the BS in the proposed mechanism is 17438.1, whereas that of the LEACH protocol is 17952.3 in all the runs with 514.2 lesser packets (approximately 3\%) transmitted to the BS in the proposed mechanism. The decreasing number of the transmitted packets to the BS in the proposed mechanism indicates that it reduces the number of the duplicated packets which are sent to the BS in the LEACH protocol, followed by decreasing the consumed energy in the

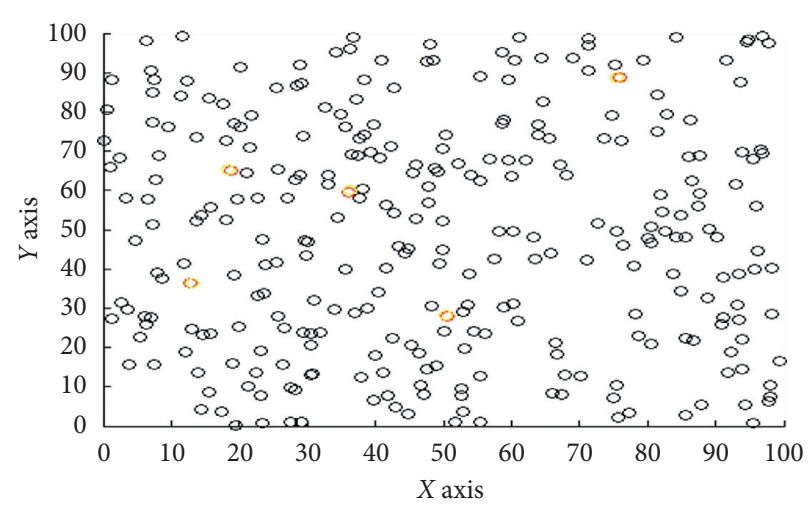

Figure 7: Adjacent sensors.

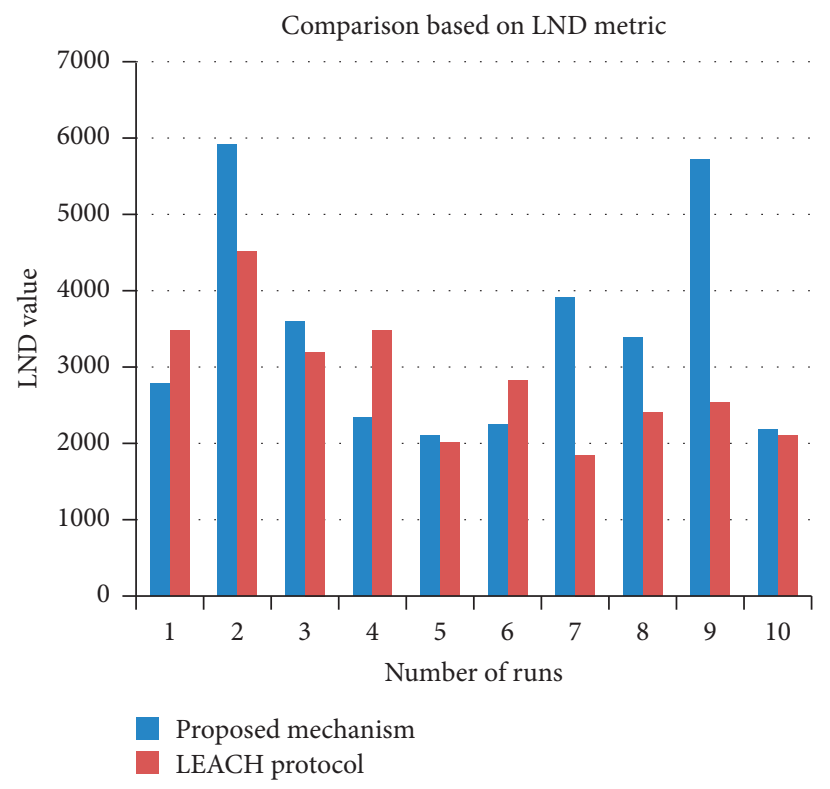

FIgURE 8: Comparison between the two mechanisms based on LND.

adjacent sensors. In the LEACH protocol, all sensed packets are sent to the $\mathrm{CH}$ by sensors, and then the $\mathrm{CH}$ sends them to the BS, and even some of these packets are duplicated. By contrast, adjacent sensors in the proposed mechanism sense different events and then send unduplicated packets to the $\mathrm{CH}$, which sends less number of packets to the BS.

Figure 10 shows the number of the transmitted packets from the sensors to the $\mathrm{CHs}$ in the proposed mechanism and the LEACH protocol.

As noted in Figure 10, the number of the transmitted packets from the sensors to the $\mathrm{CHs}$ in the proposed mechanism is 395031.6, while it is 336199.4 in the LEACH protocol with an increment of 58832.2 in the proposed 


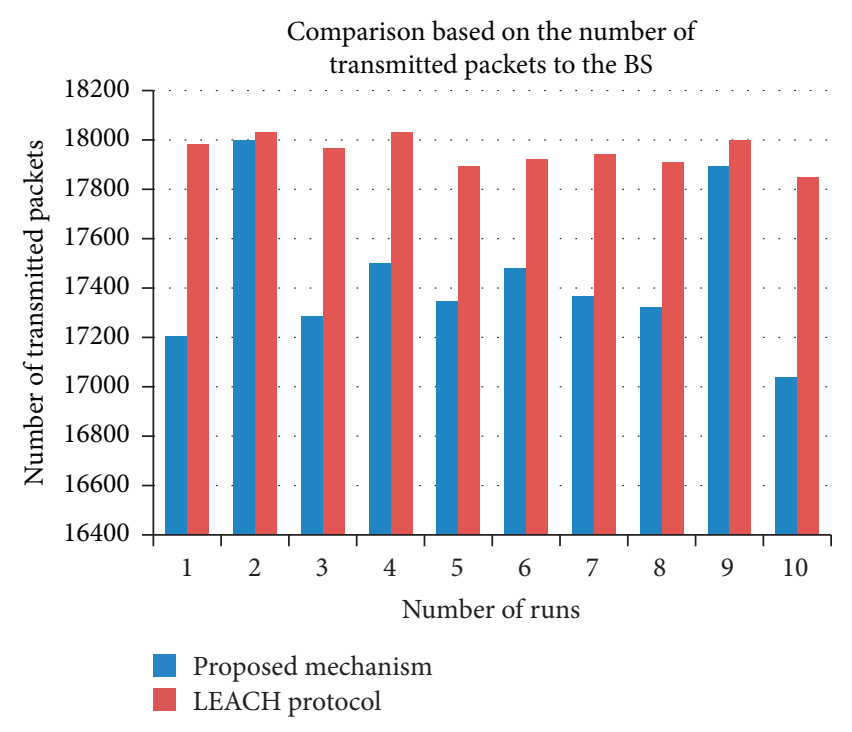

Figure 9: Comparison between the two mechanisms based on number of transmitted packets to the BS.

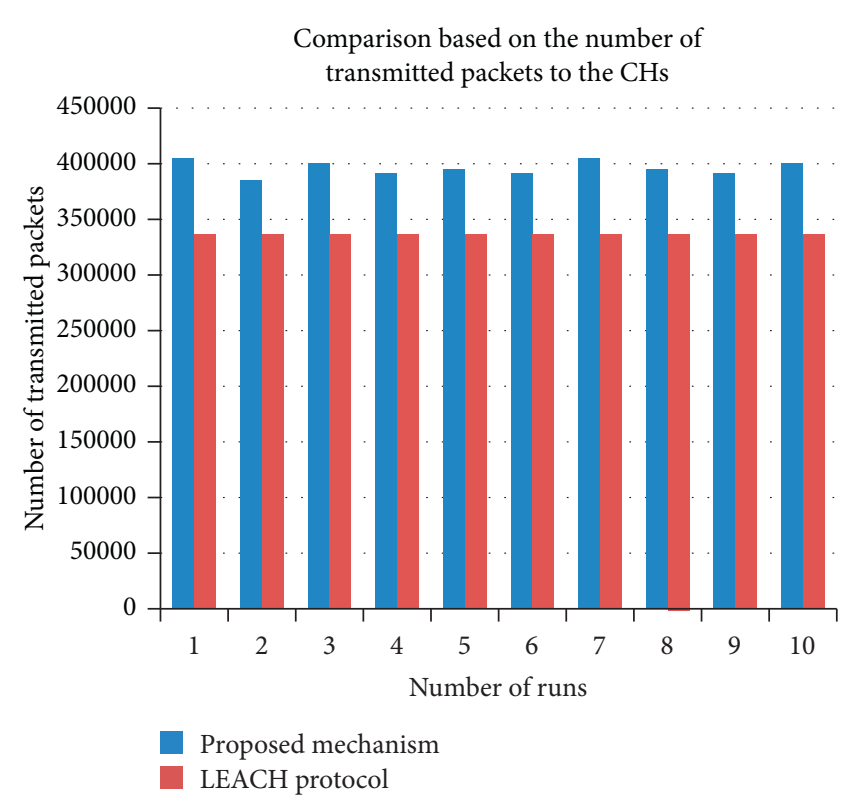

FIgURE 10: Comparison between the two mechanisms based on the number of the transmitted packets to the CHs.

mechanisms in all the runs (approximately 18\%). The increase of the transmitted packets from the sensors to the $\mathrm{CHs}$ in the proposed mechanism is due to the energy saving in the adjacent sensors as much as possible, while they keep sensing the surrounding environmental parameters longer than those in the LEACH protocol.

These simulation results present the proposed mechanism that outperforms the LEACH protocol in terms of the LND and number of transmitted packets' parameters. These significant enhancements of the proposed mechanism are due to the efficient utilization of the adjacent sensors, efficient identification of the adjacent sensors, and efficient proposal of the predefined threshold.

\section{Conclusions}

In a WSN, the distance between sensors significantly impacts its performance. Several studies have been conducted to address this problem. In this paper, a mechanism is presented to minimize the effect of the adjacent sensors. The proposed mechanism outperforms the LEACH protocol in terms of the network lifetime, the number of the transmitted packets to the BS, and the number of the transmitted packets to the $\mathrm{CHs}$. The simulation results show that the proposed mechanism increases the overall network lifetime by approximately $21 \%$ depending on the number of rounds and by approximately $18 \%$ based on the number of the transmitted packets to the CHs. Moreover, the proposed mechanism reduces the number of transmitted packets to the BS by approximately $3 \%$. These factors are important considerations in the proposed mechanism.

\section{Data Availability}

Data are available upon request to the corresponding author.

\section{Disclosure}

The sponsors do not have any role in the design of the study; in the collection, analyses, or interpretation of data; in the writing of the manuscript; or in the decision to publish the results.

\section{Conflicts of Interest}

The authors declare that there are no conflicts of interest.

\section{Acknowledgments}

The authors are grateful to the World Islamic Sciences and Education University, Amman, Jordan, for their full financial support to this research article under the motivation of the scientific research.

\section{References}

[1] D. Culler, D. Estrin, and M. Srivastava, "Guest editors' introduction: overview of sensor networks," Computer, vol. 37, no. 8, pp. 41-49, 2004.

[2] A. A. Abbasi and M. Younis, "A survey on clustering algorithms for wireless sensor networks," Computer Communications, vol. 30, no. 14-15, pp. 2826-2841, 2007.

[3] W. B. Heinzelman, A. P. Chandrakasan, and H. Balakrishnan, "An application-specific protocol architecture for wireless microsensor networks," IEEE Transactions on Wireless Communications, vol. 1, no. 4, pp. 660-670, 2002.

[4] H. Zhou, Z. Jiang, and X. Mo, "Study and design on cluster routing protocols for wireless sensor networks," 2006.

[5] W. Heinzelman, A. Chandrakasan, and H. Balakrishnan, "Energy-efficient routing protocols for wireless microsensor networks," in Proceedings of the 33rd Annual Hawaii International Conference System Sciences (HICSS), Maui, HI, USA, January 2000.

[6] M. Al-Shalabi, M. Anbar, T.-C. Wan, and K. Ahmad, "Variants of the low-energy adaptive clustering hierarchy protocol: 
survey, issues and challenges," Electronics, vol. 7, no. 8, p. 136, 2018.

[7] K. Vijayalakshmi and P. Anandan, "Global levy flight of cuckoo search with particle swarm optimization for effective cluster head selection in wireless sensor network," Intelligent Automation and Soft Computing, vol. 26, no. 2, pp. 303-311, 2020.

[8] M. Al-Shalabi, M. Anbar, T.-C. Wan, and Z. Alqattan, "Energy efficient multi-hop path in wireless sensor networks using an enhanced genetic algorithm," Information Sciences, vol. 500, pp. 259-273, 2019.

[9] A. A. Hady, "Duty cycling centralized hierarchical routing protocol with content analysis duty cycling mechanism for wireless sensor networks," Computer Systems Science And Engineering, vol. 35, no. 5, pp. 347-355, 2020.

[10] M. Al-Shalabi, M. Anbar, and T. C. Wan, "Proposed mechanism based on genetic algorithm to find the optimal multihop path in wireless sensor networks," in Proceedings of the 3rd International Conference of Reliable Information and Communication Technology, pp. 510-522, Springer, Kuala Lumpur, Malaysia, June 2018.

[11] S. Lindsey and C. Raghavendra, "Pegasis: power-efficient gathering in sensor information systems," in Proceedings of the IEEE Aerospace Conference, vol. 3, Big Sky, MT, USA, March 2002.

[12] A. Manjeshwar and D. P. Agrawal, "Teen: a routing protocol for enhanced efficiency in wireless sensor networks," in Proceedings 15th International Parallel and Distribution Processing Symposium, pp. 2009-2015, San Francisco, CA, USA, April 2001.

[13] A. Manjeshwar and D. P. Agrawal, "APTEEN: a hybrid protocol for efficient routing and comprehensive information retrieval in wireless," in Proceedings of the 16th International Parallel and Distributed Processing Symposium, p. 195, Ft. Lauderdale, FL, USA, April 2002.

[14] M. Masdari, S. M. Bazarchi, and M. Bidaki, "Analysis of secure LEACH-based clustering protocols in wireless sensor networks," Journal of Network and Computer Applications, vol. 36, pp. 1243-1260, 2013.

[15] A. Ihsan, K. Saghar, and T. Fatima, "Analysis of LEACH protocol(s) using formal verification," in Proceedings of the 2015 12th International Bhurban Conference on Applied Sciences and Technology (IBCAST), pp. 254-262, Islamabad, Pakistan, January 2015.

[16] S. Tyagi and N. Kumar, "A systematic review of clustering and routing techniques based upon LEACH protocol for wireless sensor networks," Journal of Network and Computer Applications, vol. 36, pp. 623-645, 2013.

[17] N. R. Sivakumar, "Stabilizing energy consumption in unequal clusters of wireless sensor networks," Cmc-Computers $\mathrm{Ma}$ terials and Continua, vol. 64, no. 1, pp. 81-96, 2020.

[18] M. A. Al-Shalabi, M. Anbar, and A. Obeidat, "Alternating sensing process to prolong the lifetime of wireless sensor networks," Journal of Theoretical and Applied Information Technology, vol. 97, no. 7, pp. 2132-2141, 2019, p.

[19] M. O. Al-Shalabi and M. Al-Shalabi, “An approach for MAC protocol in wireless sensor network-the group split property (GSP)," Scientific Annals of Computer Science, vol. 10, no. 1, pp. 90-93, 2012.

[20] B. Mrinmoy and S. Gupta, "Determining redundant sensors in a location unaware wireless sensor network," in Proceedings of the IEEE International Conference on Advanced Communication Control and Computing Technologies (ICACCCT 2014), Ramanathapuram, India, May 2014.
[21] T. Agrawal and R. Kushwah, "Layered clustering routing protocol with overlapping cluster heads in WSN," in Proceedings of the 2015 5th International Conference on Communication Systems and Network Technologies, Gwalior, India, April 2015.

[22] B. V. Devendra, D. Vasumathi, and N. Satyanarayana, "Exploiting common sensors in overlapped clusters for path optimization in wireless sensor networks," in Proceedings of the 2 nd International conference on computer and communication technologies, Hyderabad, India, September 2016.

[23] U. Pacharancey, A. Shaikh, P. Jadhav, and M. Madankar, "Coverage aware sleep scheduling in wireless sensor networks," International Journal of Scientific Research in Network Security and Communication (IJSRNSC), vol. 5, pp. 20-23, 2017.

[24] L. Y. Barai and M. A. Gaikwad, "Performance evaluation of LEACH protocol for wireless sensor network," International Journal of Innovative Research in Advanced Engineering (IJIRAE), vol. 1, no. 6, pp. 141-145, 2014.

[25] S. Kewei and S. Weisong, "Modeling the lifetime of wireless sensor networks," Sensor Letters, vol. 3, pp. 126-135, 2005. 